\title{
ON A DIRECT CONSTRUCTION OF INVERSE SCATTERING PROBLEMS FOR INTEGRABLE NONLINEAR EVOLUTION EQUATIONS IN THE TWO-SPATIAL DIMENSION
}

\author{
H. H. CHEN and J. E. LIN \\ Received 30 December 2003
}

\begin{abstract}
We present a method to construct inverse scattering problems for integrable nonlinear evolution equations in the two-spatial dimension. The temporal component is the adjoint of the linearized equation and the spatial component is a partial differential equation with respect to the spatial variables. Although this idea has been known for the one-spatial dimension for some time, it is the first time that this method is presented for the case of the higherspatial dimension. We present this method in detail for the Veselov-Novikov equation and the Kadomtsev-Petviashvili equation.
\end{abstract}

2000 Mathematics Subject Classification: 37K10.

1. Introduction. The search for the methods to construct inverse scattering problems for integrable nonlinear evolution equations has been an interesting subject, see $[5,9,14]$ for example, since the discovery of the soliton solutions for the Korteweg-de Vries equation $[1,2,6,7,10,11]$. It was known that the adjoint of the linearized equation could be used as the temporal component to construct an inverse scattering problem for integrable equations in the case of the one-spatial dimension [3]. In this note, we extend this concept to the case of the two-spatial dimension. Let the spatial variables be $x$ and $y$. The spatial component is assumed to be of the form $L \phi_{y}=R \phi$, where $L$ and $R$ are assumed to be partial differential operators with respect to $x$. Requiring the temporal equation and the spatial equation to be compatible, we obtain an explicit form for the spatial component as a special solution to the compatibility condition. To find the spatial component is the main work of this note. A consequence of this process is a natural derivation of a Miura-type transformation that can be used to generate Lax pairs for the integrable equations. We present it in details for the Veselov-Novikov equation [13], which is also known as the Nizhnik-Veselov-Novikov equation [12], and the Kadomtsev-Petviashvili equation [8].

\section{Veselov-Novikov equation.}

$$
u_{t}=u_{x x x}+u_{y y y}+3(u v)_{x}+3(u w)_{y},
$$

where $u$ is a function of $t, x$, and $y, v$ and $w$ depend on $u$ with $v_{y}=u_{x}, w_{x}=u_{y}$, and the subscript denotes the partial differentiation, for example, $u_{x}=\partial u / \partial x, u_{y}=$ $\partial u / \partial y, a_{1, x}=\partial a_{1} / \partial x$, and so forth. 
The adjoint of the linearized equation of (2.1) is

$$
\phi_{t}=\phi_{x x x}+\phi_{y y y}+3 v \phi_{x}+3 \Psi_{x}+3 w \phi_{y}+3 \partial_{x}^{-1} \partial_{y}\left(u \phi_{y}\right)
$$

where

$$
\Psi_{y}=u \phi_{x}
$$

We now propose

$$
L \phi_{y}=R \phi
$$

where $L$ and $R$ are partial differential operators with respect to $x$,

$$
\begin{aligned}
& L=e_{0}+e_{1} \partial_{x}+e_{2} \partial_{x}^{2}+\cdots+e_{m} \partial_{x}^{m}, \\
& R=r_{0}+r_{1} \partial_{x}+r_{2} \partial_{x}^{2}+\cdots+r_{n} \partial_{x}^{n},
\end{aligned}
$$

and where $e_{i}, 0 \leq i \leq m$, and $r_{j}, 0 \leq j \leq n$, are functions depending on $u$ and its partial derivatives but not explicitly on $t, x$, or $y$.

We request that (2.2) and (2.4) be compatible. Since (2.2) is symmetric with respect to $x$ and $y$, we also request that (2.4) be symmetric with respect to $x$ and $y$. Interchanging $x$ and $y$ in (2.4), we get

$$
\left(e_{0}^{*}+e_{1}^{*} \partial_{y}+e_{2}^{*} \partial_{y}^{2}+\cdots+e_{m}^{*} \partial_{y}^{m}\right) \phi_{x}=\left(r_{0}^{*}+r_{1}^{*} \partial_{y}+r_{2}^{*} \partial_{y}^{2}+\cdots+r_{n}^{*} \partial_{y}^{n}\right) \phi,
$$

where $e_{i}^{*}$ and $r_{j}^{*}$ are obtained from $e_{i}$ and $r_{j}, 0 \leq i \leq m, 0 \leq j \leq n$, by interchanging $x$ and $y$, respectively. In order for (2.6) to be the same as (2.4), we need $m=1$ and $n=1$. Thus (2.4) becomes

$$
\left(e_{0}+e_{1} \partial_{x}\right) \phi_{y}=\left(r_{0}+r_{1} \partial_{x}\right) \phi
$$

In order to find $e_{0}, e_{1}, r_{0}$, and $r_{1}$, we rewrite (2.7) as

$$
\phi_{y}=\left(e_{0}+e_{1} \partial_{x}\right)^{-1}\left(r_{0}+r_{1} \partial_{x}\right) \phi=\left(b_{0}+b_{-1} \partial_{x}^{-1}+b_{-2} \partial_{x}^{-2}+\cdots\right) \phi .
$$

Equating the coefficients of $\partial_{x}^{(n)} \phi, n \leq 1$, on both sides of

$$
\left(e_{0}+e_{1} \partial_{x}\right)\left(b_{0}+b_{-1} \partial_{x}^{-1}+b_{-2} \partial_{x}^{-2}+\cdots\right) \phi=\left(r_{0}+r_{1} \partial_{x}\right) \phi,
$$

we get

$$
\begin{gathered}
e_{1} b_{0}=r_{1}, \\
e_{1} b_{0, x}+e_{0} b_{0}+e_{1} b_{-1}=r_{0}, \\
e_{1} b_{-1, x}+e_{0} b_{-1}+e_{1} b_{-2}=0, \\
e_{1} b_{-h, x}+e_{0} b_{-h}+e_{1} b_{-h-1}=0, \quad h \geq 1 .
\end{gathered}
$$

Now back to (2.3), $\Psi$ must be expressible in terms of $\phi$. Therefore, we propose

$$
\Psi=\left(a_{k} \partial_{x}^{k}+a_{k-1} \partial_{x}^{k-1}+\cdots\right) \phi .
$$


Substituting it into (2.3), we get

$$
\Psi_{y}=a_{k, y} \partial_{x}^{k} \phi+a_{k} \partial_{x}^{k} \phi_{y}+a_{k-1, y} \partial_{x}^{k-1} \phi+a_{k-1} \partial_{x}^{k-1} \phi_{y}+\cdots=u \phi_{x} .
$$

Substituting $\phi_{y}$ by (2.8) into (2.15) and equating the coefficients of $\partial_{x}^{(n)} \phi$ on both sides of (2.15), we get $k=1$. Thus

$$
\Psi=\left(a_{1} \partial_{x}+a_{0}+a_{-1} \partial_{x}^{-1}+\cdots\right) \phi .
$$

Furthermore, equating the coefficients of $\partial_{x}^{(n)} \phi$ on both sides of (2.15) with $k=1$ for $n \leq 1$, we get, for the coefficient of $\phi_{x}$,

$$
a_{1, y}+a_{1} b_{0}=u
$$

for the coefficient of $\phi$,

$$
a_{1} b_{0, x}+a_{1} b_{-1}+a_{0, y}+a_{0} b_{0}=0,
$$

for the coefficient of $\partial_{x}^{-1} \phi$,

$$
a_{1} b_{-1, x}+a_{1} b_{-2}+a_{0} b_{-1}+a_{-1, y}+a_{-1} b_{0}=0,
$$

for the coefficient of $\partial_{x}^{-2} \phi$,

$$
a_{1} b_{-2, x}+a_{1} b_{-3}+a_{0} b_{-2}-a_{-1} b_{0, x}+a_{-1} b_{-1}+a_{-2, y}+a_{-2} b_{0}=0,
$$

and for the coefficient of $\partial_{x}^{-h} \phi$,

$$
a_{1} b_{-h, x}+a_{1} b_{-h-1}+a_{0} b_{-h}+\left(\text { terms linear in } a_{-1}, \ldots, a_{-h+1}\right)+a_{-h, y}+a_{-h} b_{0}=0,
$$

for $h \geq 2$.

We now demand that (2.2) and (2.8) be compatible, that is, $\phi_{t x y}=\phi_{y t x}$.

Differentiating both sides of (2.2) with respect to $x$ and $y$, we get

$$
\begin{aligned}
\phi_{t x y}= & \phi_{x x x x y}+\phi_{y y y x y}+3 v_{x y} \phi_{x}+3 v_{x} \phi_{x y}+3 v_{y} \phi_{x x}+3 v \phi_{x x y} \\
& +3 \Psi_{x x y}+3 w_{x y} \phi_{y}+3 w_{x} \phi_{y y}+3 w_{y} \phi_{x y} \\
& +3 w \phi_{x y y}+3 u_{y y} \phi_{y}+6 u_{y} \phi_{y y}+3 u \phi_{y y y} .
\end{aligned}
$$

Differentiating both sides of (2.8) with respect to $t$ and $x$, we get

$$
\phi_{y t x}=\sum_{j \leq 0}\left(b_{j, t x} \partial_{x}^{j} \phi+b_{j, t} \partial_{x}^{j+1} \phi+b_{j, x} \partial_{x}^{j} \phi_{t}+b_{j} \partial_{x}^{j+1} \phi_{t}\right) .
$$

By equating the coefficients of $\partial_{x}^{(n)} \phi$ on both sides of $\phi_{t x y}=\phi_{y t x}$ after we use (2.2), (2.8), and (2.16) to express both sides in terms of $\partial_{x}^{(n)} \phi$, we get, for the coefficient of $\partial_{x}^{4} \phi$,

$$
b_{0}=b_{0} \text {, }
$$


for the coefficient of $\partial_{x}^{3} \phi$,

$$
u=-b_{0, x}+b_{0} a_{1}
$$

for the coefficient of $\partial_{x}^{2} \phi$,

$$
2 b_{0, x x}+b_{-1, x}+v_{y}+2 u_{x}=b_{0, x} a_{1}+2 b_{0} a_{1, x}+b_{0} a_{0}+b_{-1} a_{1},
$$

and for the coefficient of $\partial_{x} \phi$,

$$
\begin{aligned}
4 b_{0, x x x} & +6 b_{-1, x x}+3 b_{-2, x}+b_{0, y y y}+4 b_{0, y y} b_{0}+3\left(b_{0, y}\right)^{2}+6 b_{0, y} b_{0}^{2}+3 v_{x y} \\
& +3 v b_{0, x}+3 u_{x x}+3 w_{y} b_{0}+3 w b_{0, y} \\
= & b_{0, t}+3 b_{0, x}\left(a_{1, x}+a_{0}\right)+b_{0} b_{0, y y}+3 b_{0, y} b_{0}^{2}+3 b_{0}\left(a_{1, x x}+2 a_{0, x}+a_{-1}\right) \\
& +3 b_{-1, x} a_{1}+3 b_{-1}\left(a_{1, x}+a_{0}\right)+3 b_{-2} a_{1} .
\end{aligned}
$$

From (2.17) and (2.25), we get $a_{1, y}=-b_{0, x}$. Let $q=-\partial_{x}^{-1} a_{1}$, then

$$
a_{1}=-q_{x}, \quad b_{0}=q_{y},
$$

and, from (2.25),

$$
u=-q_{x y}-q_{x} q_{y} .
$$

Substituting (2.29) into (2.1), we get

$$
\begin{aligned}
0 & =u_{t}-u_{x x x}-u_{y y y}-3(u v)_{x}-3(u w)_{y} \\
& =M\left(q_{t}-q_{x x x}-q_{y y y}-3 q_{x x} q_{x}-q_{x}^{3}-3 v q_{x}-3 q_{y y} q_{y}-q_{y}^{3}-3 w q_{y}\right),
\end{aligned}
$$

where $M=-q_{y} \partial_{x}-q_{x} \partial_{y}-\partial_{x y}^{2}$.

Thus (2.29) is a Miura-type transformation relating the Veselov-Novikov equation, (2.1), to the modified Veselov-Novikov equation

$$
q_{t}-q_{x x x}-q_{y y y}-3 q_{x x} q_{x}-q_{x}^{3}-3 v q_{x}-3 q_{y y} q_{y}-q_{y}^{3}-3 w q_{y}=0 .
$$

From (2.18), we get

$$
b_{-1}=\left(\frac{-1}{a_{1}}\right)\left(a_{1} b_{0, x}+a_{0, y}+a_{0} b_{0}\right) .
$$

Note that $a_{1} \neq 0$ from (2.17).

Substituting (2.32) into (2.26) and using (2.28) and (2.29), we get

$$
\left(q_{x} \partial_{x y}^{2}+\left(-q_{x x}+q_{x}^{2}\right) \partial_{y}+q_{x} q_{y} \partial_{x}+q_{x} q_{x y}-q_{y} q_{x x}\right)\left(a_{0}-q_{x}^{2}\right)=0 .
$$

We take

$$
a_{0}=q_{x}^{2}
$$


as it is an obvious solution to the above equation. Therefore, from (2.32), we have

$$
b_{-1}=q_{x y}+q_{x} q_{y}=-u
$$

Now, from (2.19), we have

$$
b_{-2}=\left(\frac{-1}{a_{1}}\right)\left(a_{1} b_{-1, x}+a_{0} b_{-1}+a_{-1, y}+a_{-1} b_{0}\right) .
$$

Substituting it into (2.27) and using (2.28), (2.29), (2.31), (2.34), and (2.35), we get

$$
\left[\partial_{y}+\partial_{x}\left(\left(\frac{1}{q_{x}}\right)\left(\partial_{y}+q_{y}\right)\right)\right] a_{-1}=0
$$

Thus we take

$$
a_{-1}=0
$$

as an obvious solution. Therefore, (2.36) becomes

$$
b_{-2}=-q_{x x y}-q_{x x} q_{y}+q_{x}^{2} q_{y} .
$$

We are now ready to solve (2.7). Normalizing $e_{1}=1$, we have, from (2.10),

$$
r_{1}=b_{0}=q_{y} .
$$

From (2.12) and (2.39), we have

$$
\begin{aligned}
e_{0}\left(q_{x y}+q_{x} q_{y}\right) & =-\left(q_{x y}+q_{x} q_{y}\right)_{x}-\left(-q_{x x y}-q_{x x} q_{y}+q_{x}^{2} q_{y}\right) \\
& =-q_{x}\left(q_{x y}+q_{x} q_{y}\right) .
\end{aligned}
$$

Hence $e_{0}=-q_{x}$, since $q_{x y}+q_{x} q_{y}=-u \neq 0$. From (2.11), we have

$$
r_{0}=q_{x y}+\left(-q_{x}\right) q_{y}+q_{x y}+q_{x} q_{y}=2 q_{x y} .
$$

Hence, (2.7) becomes

$$
\left(\partial_{x}-q_{x}\right) \phi_{y}=\left(q_{y} \partial_{x}+2 q_{x y}\right) \phi
$$

Thus (2.2) and (2.43) form a pair of inverse scattering problems for (2.1).

REMARK 2.1. Since $v_{y}=u_{x}, w_{x}=u_{y}$, and $u=-q_{x y}-q_{x} q_{y}$, we have

$$
\begin{gathered}
v=-q_{x x}-\partial_{y}^{-1}\left(q_{x x} q_{y}\right)-\left(\frac{1}{2}\right) q_{x}^{2}, \\
w=-q_{y y}-\partial_{x}^{-1}\left(q_{x} q_{y y}\right)-\left(\frac{1}{2}\right) q_{y}^{2} .
\end{gathered}
$$

Substituting $u, v$, and $w$ by their expressions in terms of $q$ into (2.2), and using (2.43), we get an inverse scattering problem for the modified Veselov-Novikov equation, (2.31). 
REMARK 2.2. From (2.13), we have

$$
b_{-h, x}-q_{x} b_{-h}+b_{-h-1}=0, \quad h \geq 1 .
$$

Multiplying it by $a_{1}$,

$$
a_{1} b_{-h, x}-a_{1} q_{x} b_{-h}+a_{1} b_{-h-1}=0 .
$$

Since $a_{0}=-a_{1} q_{x}$,

$$
a_{1} b_{-h, x}+a_{0} b_{-h}+a_{1} b_{-h-1}=0 .
$$

Now, suppose $a_{-1}=\cdots=a_{-h+1}=0$, then from (2.21) and (2.47),

$$
\left(a_{-h}\right)_{y}+a_{-h} b_{0}=0
$$

that is,

$$
\left(\partial_{y}+q_{y}\right) a_{-h}=0
$$

Thus we can take $a_{-h}=0$. Since we have shown in (2.38) that we can take $a_{-1}=0$, hence we can take $a_{-h}=0, h \geq 1$. Note that this provides a consistency between (2.13) and (2.21). Also, (2.14) becomes $\Psi=\left(a_{1} \partial_{x}+a_{0}\right) \phi=-q_{x} \phi_{x}+q_{x}^{2} \phi$.

REMARK 2.3. From (2.47),

$$
\begin{aligned}
b_{-h-1} & =-b_{-h, x}+q_{x} b_{-h}=\left(-\partial_{x}+q_{x}\right) b_{-h} \\
& =\left(-\partial_{x}+q_{x}\right)^{h} b_{-1}=-\left(-\partial_{x}+q_{x}\right)^{h} u, \quad \text { for } h \geq 1 .
\end{aligned}
$$

This provides a recursive relation among the coefficients of (2.8).

REMARK 2.4. Let $q=\ln \Psi$; we get from (2.29) and (2.31) that

$$
\Psi_{x y}+u \Psi=0, \quad \Psi_{t}=\Psi_{x x x}+3 v \Psi_{x}+\Psi_{y y y}+3 w \Psi_{y}
$$

This is the inverse scattering problem for (2.1) given in [13].

3. Kadomtsev-Petviashvili equation. We now turn to the Kadomtsev-Petviashvili equation [8]

$$
u_{t}=\partial_{x}^{-1} u_{y y}-u_{x x x}-6 u u_{x}
$$


The adjoint of its linearized equation is

$$
\phi_{t}=\partial_{x}^{-1} \phi_{y y}-\phi_{x x x}-6 u \phi_{x}
$$

Just like in the case of the Veselov-Novikov equation, we propose (2.4) where $L$ and $R$ are partial differential operators with respect to $x$ and the coefficients depend on $u$ and its partial derivatives but not explicitly on $x, y$, or $t$.

We demand that (3.2) and (2.4) be compatible. In order to do so, we rewrite (2.4) as $\phi_{y}=L^{-1} R \phi$, where, formally,

$$
L^{-1} R=a_{m} \partial_{x}^{m}+a_{m-1} \partial_{x}^{m-1}+\cdots+a_{0}+a_{-1} \partial_{x}^{-1}+\cdots=\sum_{k \leq m} a_{k} \partial_{x}^{k}
$$

Thus

$$
\phi_{y}=\sum_{k \leq m} a_{k} \partial_{x}^{k} \phi
$$

We now demand that (3.2) and (3.4) be compatible, that is, $\phi_{t x y}=\phi_{y t x}$. Differentiating both sides of (3.2) with respect to $x$ and $y$, we get

$$
\begin{aligned}
\phi_{t x y}= & \sum_{k \leq m} a_{k, y y} \partial_{x}^{k} \phi+2 \sum_{k \leq m} a_{k, y} \partial_{x}^{k} \phi_{y}+\sum_{k \leq m} a_{k} \partial_{x}^{k} \phi_{y y}-6 u_{x y} \phi_{x} \\
& -6 u_{x}\left(\sum_{k \leq m} a_{k, x} \partial_{x}^{k} \phi+\sum_{k \leq m} a_{k} \partial_{x}^{k+1} \phi\right)-6 u_{y} \phi_{x x} \\
& -6 u\left(\sum_{k \leq m} a_{k, x x} \partial_{x}^{k} \phi+2 \sum_{k \leq m} a_{k, x} \partial_{x}^{k+1} \phi+\sum_{k \leq m} a_{k} \partial_{x}^{k+2} \phi\right) \\
& -\left(\sum_{k \leq m} a_{k, x x x x} \partial_{x}^{k} \phi+4 \sum_{k \leq m} a_{k, x x x} \partial_{x}^{k+1} \phi+6 \sum_{k \leq m} a_{k, x x} \partial_{x}^{k+2} \phi\right. \\
& \left.+4 \sum_{k \leq m} a_{k, x} \partial_{x}^{k+3} \phi+\sum_{k \leq m} a_{k} \partial_{x}^{k+4} \phi\right) .
\end{aligned}
$$

Differentiating both sides of (3.4) with respect to $t$ and $x$, we get

$$
\begin{aligned}
\phi_{y t x}= & a_{m, t} \partial_{x}^{m+1} \phi+\sum_{k \leq m}\left(a_{k, t x}+a_{k-1, t}\right) \partial_{x}^{k} \phi \\
& +\sum_{k \leq m} a_{k} \partial_{x}^{k} \phi_{y y}-6 \sum_{k \leq m} a_{k} \partial_{x}^{k+1}\left(u \phi_{x}\right)-\sum_{k \leq m} a_{k} \partial_{x}^{k+4} \phi \\
& +\sum_{k \leq m} a_{k, x} \partial_{x}^{k-1} \phi_{y y}-6 \sum_{k \leq m} a_{k, x} \partial_{x}^{k}\left(u \phi_{x}\right)-\sum_{k \leq m} a_{k, x} \partial_{x}^{k+3} \phi .
\end{aligned}
$$


Demanding $\phi_{y t x}=\phi_{t x y}$, we get, after simplification,

$$
\begin{aligned}
a_{m, t} \partial_{x}^{m+1} \phi & +\sum_{k \leq m}\left(a_{k, t x}+a_{k-1, t}\right) \partial_{x}^{k} \phi-6 \sum_{k \leq m} a_{k} \partial_{x}^{k+1}\left(u \phi_{x}\right)+\sum_{k \leq m} a_{k, x} \partial_{x}^{k-1} \phi y y \\
& -6 \sum_{k \leq m} a_{k, x} \partial_{x}^{k}\left(u \phi_{x}\right)+3 \sum_{k \leq m} a_{k, x} \partial_{x}^{k+3} \phi \\
= & \sum_{k \leq m} a_{k, y} \partial_{x}^{k} \phi+2 \sum_{k \leq m} a_{k, y} \partial_{x}^{k} \phi_{y}-6 u_{x y} \phi_{x} \\
& -6 u_{x}\left(\sum_{k \leq m} a_{k, x} \partial_{x}^{k} \phi+\sum_{k \leq m} a_{k} \partial_{x}^{k+1} \phi\right)-6 u_{y} \phi_{x x} \\
& -6 u\left(\sum_{k \leq m} a_{k, x x} \partial_{x}^{k} \phi+2 \sum_{k \leq m} a_{k, x} \partial_{x}^{k+1} \phi+\sum_{k \leq m} a_{k} \partial_{x}^{k+2} \phi\right) \\
& -\left(\sum_{k \leq m} a_{k, x x x x} \partial_{x}^{k} \phi+4 \sum_{k \leq m} a_{k, x x x} \partial_{x}^{k+1} \phi+6 \sum_{k \leq m} a_{k, x x} \partial_{x}^{k+2} \phi\right) .
\end{aligned}
$$

ClaIm 3.1 (if $m \geq 3$, then $a_{m}=0$ ). Suppose $m \geq 3$ and $a_{m} \neq 0$. Using (3.4) for $\phi_{y}$ and $\phi_{y y}$ to equate the coefficients of the same order of partial derivatives of $\phi$ with respect to $x$ on both sides of (3.7), we get, for the coefficient of $\partial_{x}^{3 m-1} \phi: a_{m, x} a_{m}^{2}=0$. Hence,

$$
a_{m}=\text { constant } \neq 0 \text {. }
$$

Note that since $m \geq 3, \partial_{x}^{3 m-1} \phi$ is the highest order of partial derivative of $\phi$ with respect to $x$. Substituting (3.8) into (3.7), we get

$$
\begin{aligned}
& a_{m-1, t} \partial_{x}^{m} \phi+\sum_{k \leq m-1}\left(a_{k, t x}+a_{k-1, t}\right) \partial_{x}^{k} \phi-6 \sum_{k \leq m} a_{k} \partial_{x}^{k+1}\left(u \phi_{x}\right) \\
& \quad+\sum_{k \leq m-1} a_{k, x} \partial_{x}^{k-1} \phi_{y y}-6 \sum_{k \leq m-1} a_{k, x} \partial_{x}^{k}\left(u \phi_{x}\right)+3 \sum_{k \leq m-1} a_{k, x} \partial_{x}^{k+3} \phi \\
&= \sum_{k \leq m-1} a_{k, y y} \partial_{x}^{k} \phi+2 \sum_{k \leq m-1} a_{k, y} \partial_{x}^{k} \phi_{y}-6 u_{x y} \phi_{x} \\
&-6 u_{x}\left(\sum_{k \leq m-1} a_{k, x} \partial_{x}^{k} \phi+\sum_{k \leq m} a_{k} \partial_{x}^{k+1} \phi\right)-6 u_{y} \phi_{x x} \\
&-6 u\left(\sum_{k \leq m-1} a_{k, x x} \partial_{x}^{k} \phi+2 \sum_{k \leq m-1} a_{k, x} \partial_{x}^{k+1} \phi+\sum_{k \leq m} a_{k} \partial_{x}^{k+2} \phi\right) \\
&-\left(\sum_{k \leq m-1} a_{k, x x x x} \partial_{x}^{k} \phi+4 \sum_{k \leq m-1} a_{k, x x x} \partial_{x}^{k+1} \phi+6 \sum_{k \leq m-1} a_{k, x x} \partial_{x}^{k+2} \phi\right) .
\end{aligned}
$$

Again, using (3.4) for $\phi_{y}$ and $\phi_{y y}$ to equate the coefficients of the partial derivatives of $\phi$ with respect to $x$ on both sides of (3.9), we get, for the coefficient of $\partial_{x}^{3 m-2} \phi$, $a_{m-1, x} a_{m}^{2}=0$. Hence $a_{m-1, x}=0, a_{m-1}=$ constant. Note that since $m \geq 3, \partial_{x}^{3 m-2} \phi$ is the highest order of partial derivatives of $\phi$ with respect to $x$ in (3.9). 
Repeating the above argument, we can show that, for the coefficient of $\partial_{x}^{3 m-h} \phi$, $a_{m-h+1, x} a_{m}^{2}=0$ for $1 \leq h<2 m-2$. Hence $a_{m-h+1, x}=0, a_{m-h+1}=$ constant for $1 \leq$ $h<2 m-2$. When $h=2 m-2, \partial_{x}^{3 m-h} \phi=\partial_{x}^{m+2} \phi$. Now, equating the coefficient of $\partial_{x}^{m+2} \phi$ on both sides of (3.9), we get $-6 a_{m} u-a_{-m+3, x} a_{m}^{2}=-6 u a_{m}$. Hence $a_{-m+3, x}=0$, $a_{-m+3}=$ constant. Now, equating the coefficient of $\partial_{x}^{m+1} \phi$ on both sides of (3.9), we get $-6 a_{m}(m+1) u_{x}-6 a_{m-1} u=6 u_{x} a_{m}-6 u a_{m-1}$. Hence $6 a_{m}(m+2) u_{x}=0$.

Hence $a_{m}=0$. But this is a contradiction to the assumption that $a_{m} \neq 0$.

Thus, if $m \geq 3$, then $a_{m}=0$. Therefore, (3.4) becomes

$$
\phi_{y}=\sum_{k \leq 2} a_{k} \partial_{x}^{k} \phi
$$

Now with $m=2$ in (3.7) and using (3.10) to equate the coefficients of $\partial_{x}^{(h)} \phi$ on both sides of (3.7) for $h \leq 5$, we get, for $\partial_{x}^{5} \phi$,

$$
\left.\left(a_{2}^{2}+3\right) a_{2, x}=0 \quad \text { (hence either } a_{2}^{2}+3=0 \text { or } a_{2}=\text { constant }\right) .
$$

For $\partial_{x}^{4} \phi$,

$$
\left(a_{2}^{2}+3\right) a_{1, x}=0 \quad \text { (hence either } a_{2}^{2}+3=0 \text { or } a_{1}=\text { constant). }
$$

For $\partial_{x}^{3} \phi$,

$$
12 a_{2} u_{x}-2 a_{1, x} a_{2} a_{1}-6 a_{1, x x}+2 a_{1, y} a_{2}-\left(a_{2}^{2}+3\right) a_{0, x}=0 .
$$

For $\partial_{x}^{2} \phi$,

$$
\begin{aligned}
a_{1, t}- & 18 a_{2} u_{x x}-6 a_{1} u_{x}+2 a_{1, x} a_{0} a_{2}+2\left(a_{1, x}\right)^{2} a_{2} \\
& +a_{1}^{2} a_{1, x}+2 a_{1} a_{2} a_{0, x}+6 a_{1, x} u-2 a_{1, y} a_{1} \\
& +6 u_{y}-2 a_{0, y} a_{2}+4 a_{1, x x x}+6 a_{0, x x}+\left(a_{2}^{2}+3\right) a_{-1, x}=0 .
\end{aligned}
$$

In view of (3.11) and (3.12), we will take $a_{2}^{2}+3=0$ so that $a_{1}$ does not have to be a constant. Without loss of generality, let $a_{2}=-(3)^{1 / 2} i$, where $i=(-1)^{1 / 2}$. From (3.13),

$$
12 a_{2} u_{x}=2 a_{1, x} a_{2} a_{1}+6 a_{1, x x}-2 a_{1, y} a_{2}
$$

Hence

$$
u_{x}=\left(\frac{1}{6}\right) a_{1, x} a_{1}+\left(\frac{1}{\left(2 a_{2}\right)}\right) a_{1, x x}-\left(\frac{1}{6}\right) a_{1, y} .
$$

Thus

$$
u=\left(\frac{1}{12}\right) a_{1}^{2}+\left(\frac{1}{\left(2 a_{2}\right)}\right) a_{1, x}-\left(\frac{1}{6}\right) \partial_{x}^{-1} a_{1, y}+\lambda \text { for some constant } \lambda .
$$


Substituting (3.17) into (3.1), we get

$$
\begin{aligned}
0 & =u_{t}-\partial_{x}^{-1} u_{y y}+u_{x x x}+6 u u_{x} \\
& =M\left(a_{1, t}-\partial_{x}^{-1} a_{1, y y}+a_{1, x x x}+\left(\frac{1}{2}\right) a_{1}^{2} a_{1, x}-\left(\partial_{x}^{-1} a_{1, y}\right) a_{1, x}+6 \lambda a_{1, x}\right),
\end{aligned}
$$

where $M=(1 / 6) a_{1}+\left(1 /\left(2 a_{2}\right)\right) \partial_{x}-(1 / 6) \partial_{x}^{-1} \partial_{y}$.

Thus (3.17) is a Miura-type transformation relating the Kadomtsev-Petviashvili equation, (3.1), to the modified Kadomtsev-Petviashvili equation

$$
a_{1, t}-\partial_{x}^{-1} a_{1, y y}+a_{1, x x x}+\left(\frac{1}{2}\right) a_{1}^{2} a_{1, x}-\left(\partial_{x}^{-1} a_{1, y}\right) a_{1, x}+6 \lambda a_{1, x}=0 .
$$

Substituting (3.17) and (3.19) into (3.14), we get

$$
K\left(a_{0}-a_{1, x}\right)=0
$$

where $K=6 \partial_{x}^{2}+2 a_{1} a_{2} \partial_{x}+2 a_{1, x} a_{2}-2 a_{2} \partial_{y}$.

Thus $a_{0}-a_{1, x}=0$ is a solution to (3.20). Hence, we take

$$
a_{0}=a_{1, x}
$$

Now equating the coefficient of $\partial_{x} \phi$ on both sides of (3.7) with the use of (3.17), (3.19), and (3.21), we get $K\left(a_{-1}\right)=0$. Again we take

$$
a_{-1}=0
$$

as the solution. Now equating the coefficient of $\phi$ on both sides of (3.7) with the use of (3.17), (3.19), (3.21), and (3.22), we get $K\left(a_{-2}\right)=0$. Again, we take $a_{-2}=0$ as the solution.

We now claim that we can take $a_{-n}=0$ for all $n \geq 1$. Assume that $a_{-h}=0$ for $1 \leq h \leq n+1$, where $n \geq 1$. We want to show that we can take $a_{-n-2}=0$ as a solution.

Equating the coefficient of $\partial_{x}^{-n} \phi$ on both sides of (3.7) and using (3.17), (3.19), and (3.21) and the assumption that $a_{-h}=0$ for $1 \leq h \leq n+1$, where $n \geq 1$, we get $K\left(a_{-n-2}\right)$ $=0$. Thus we can take $a_{-n-2}=0$ as a solution. Since we have already shown that we can take $a_{-1}=0$ and $a_{-2}=0$, we can take $a_{-n}=0$ for all $n \geq 1$. Hence (3.4) can be terminated at $a_{0}$, that is,

$$
\phi_{y}=a_{2} \phi_{x x}+a_{1} \phi_{x}+a_{0} \phi
$$

where $a_{2}=-(3)^{1 / 2} i, a_{0}=a_{1, x}$, and $a_{1}$ satisfies (3.17) and (3.19). Thus (3.2) and (3.23),

$$
\begin{aligned}
\phi_{t} & =\partial_{x}^{-1} \phi_{y y}-\phi_{x x x}-6 u \phi_{x}, \\
\phi_{y} & =a_{2} \phi_{x x}+a_{1} \phi_{x}+a_{0} \phi,
\end{aligned}
$$

form a pair of inverse scattering problems for (3.1). 
REMARK 3.2. Substituting $u=(1 / 12) a_{1}^{2}+\left(1 /\left(2 a_{2}\right)\right) a_{1, x}-(1 / 6) \partial_{x}^{-1} a_{1, y}+\lambda$ into (3.2), and using (3.23), we get a pair of inverse scattering problems for the modified Kadomtsev-Petviashvili equation, (3.19).

REMARK 3.3. Let $a_{1}=2(3)^{1 / 2} i \Psi_{x} / \Psi$. With $a_{2}=-(3)^{1 / 2} i$, we get, from (3.17) and (3.19),

$$
\begin{gathered}
\Psi_{x x}+(3)^{-1 / 2} i \Psi_{y}+u \Psi=\lambda \Psi \\
\Psi_{t}+4 \Psi_{x x x}+6 u \Psi_{x}+3 u_{x} \Psi-(3)^{1 / 2}\left(\partial_{x}^{-1} u_{y}\right) \Psi=0 .
\end{gathered}
$$

These are the Lax-pair equations found by Dryuma [4].

4. Conclusion. We have demonstrated a method to construct the inverse scattering problems for the integrable nonlinear evolution equations in the two-spatial dimension in which the temporal equation is the adjoint of the linearized equation. A Miura-type transformation and a modified equation are obtained as a byproduct of the process. An inverse scattering problem for the modified equation is also found. The applicability of this method for testing the integrability of nonlinear evolution equations in the twospatial dimension is currently under study.

\section{REFERENCES}

[1] M. J. Ablowitz and P. A. Clarkson, Solitons, Nonlinear Evolution Equations and Inverse Scattering, London Mathematical Society Lecture Note Series, vol. 149, Cambridge University Press, Cambridge, 1991.

[2] M. J. Ablowitz and H. Segur, Solitons and the Inverse Scattering Transform, SIAM Studies in Applied Mathematics, vol. 4, Society for Industrial and Applied Mathematics (SIAM), Pennsylvania, 1981.

[3] H. H. Chen, Y. C. Lee, and C. S. Liu, Integrability of nonlinear Hamiltonian systems by inverse scattering method, Phys. Scripta 20 (1979), no. 3-4, 490-492.

[4] V. Dryuma, Analytic solution of the two-dimensional Korteweg-deVries equation, JETP Lett. 19 (1974), 387-388.

[5] A. S. Fokas and V. E. Zakharov (eds.), Important Developments in Soliton Theory, Springer Series in Nonlinear Dynamics, Springer-Verlag, Berlin, 1993.

[6] C. S. Gardner, J. M. Greene, M. D. Kruskal, and R. M. Miura, Method for solving the KortewegdeVries equation, Phys. Rev. Lett. 19 (1967), 1095-1097.

[7] _ The Korteweg-de Vries equation and generalizations. VI. Methods for exact solution, Comm. Pure Appl. Math. 27 (1974), 97-133.

[8] B. B. Kadomtsev and V. I. Petviashvili, On the stability of solitary waves in weakly dispersing media, Soviet Phys. Dokl. 15 (1970), 539-541.

[9] Y. Kosmann-Schwarzbach, B. Grammaticos, and K. M. Tamizhmani (eds.), Integrability of Nonlinear Systems, Lecture Notes in Physics, vol. 495, Springer-Verlag, Berlin, 1997.

[10] P. D. Lax, Integrals of nonlinear equations of evolution and solitary waves, Comm. Pure Appl. Math. 21 (1968), 467-490.

[11] A. C. Newell, Solitons in Mathematics and Physics, CBMS-NSF Regional Conference Series in Applied Mathematics, vol. 48, Society for Industrial and Applied Mathematics (SIAM), Pennsylvania, 1985.

[12] C. Rogers, B. G. Konopelchenko, M. P. Stallybrass, and W. K. Schief, The Nizhnik-VeselovNovikov equation: associated boundary value problems, Internat. J. Non-Linear Mech. 31 (1996), no. 4, 441-450. 
[13] A. P. Veselov and S. P. Novikov, Finite-zone, two-dimensional, potential Schrödinger operators. Explicit formulas and evolution equations, Soviet Math. Dokl. 30 (1984), 588591.

[14] V. E. Zakharov (ed.), What Is Integrability? Springer Series in Nonlinear Dynamics, SpringerVerlag, Berlin, 1991.

H. H. Chen: Department of Physics and Astronomy, University of Maryland, College Park, MD 20742, USA

E-mail address: chenhh@umd. edu

J. E. Lin: Department of Mathematical Sciences, George Mason University, Fairfax, VA 22030, USA

E-mail address: je1in@gmu.edu 


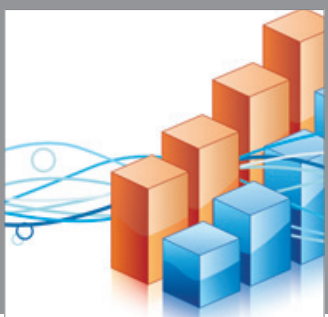

Advances in

Operations Research

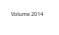

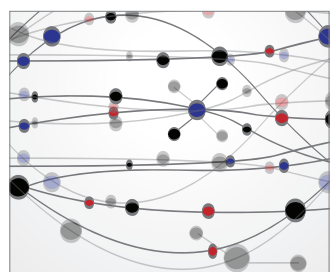

\section{The Scientific} World Journal
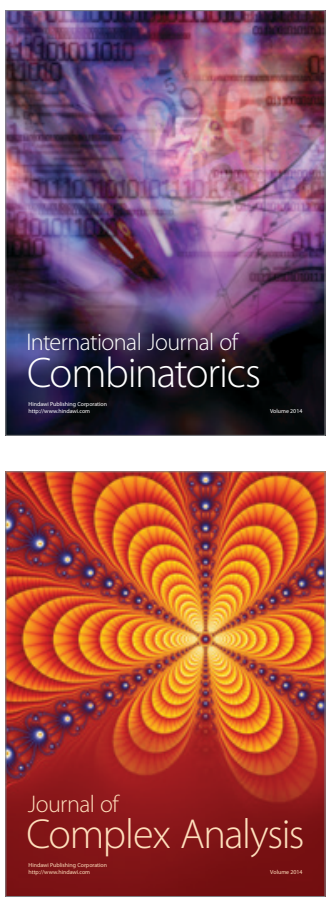

International Journal of

Mathematics and

Mathematical

Sciences
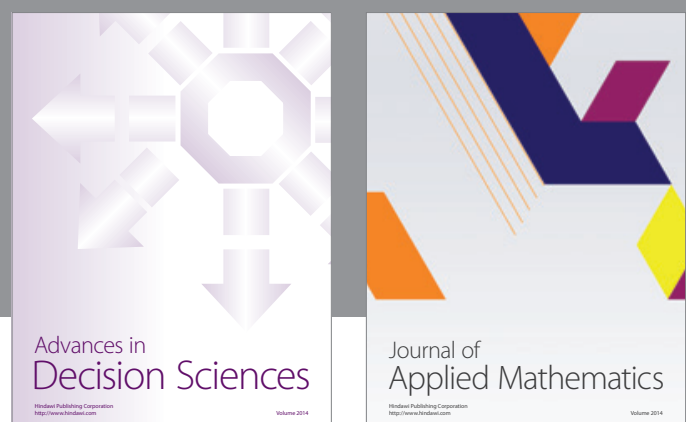

Journal of

Applied Mathematics
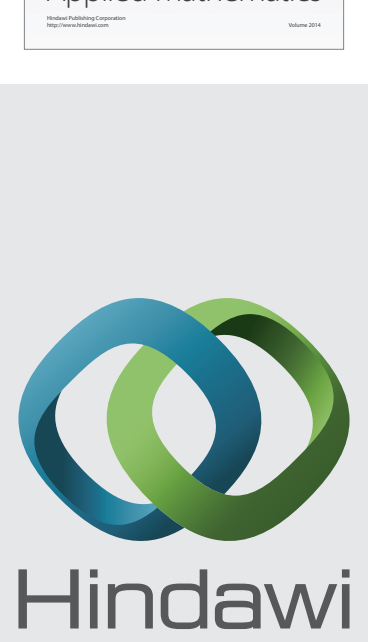

Submit your manuscripts at http://www.hindawi.com
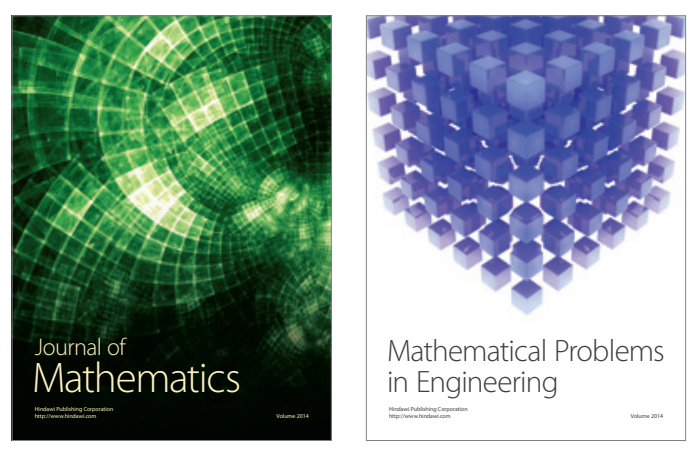

Mathematical Problems in Engineering
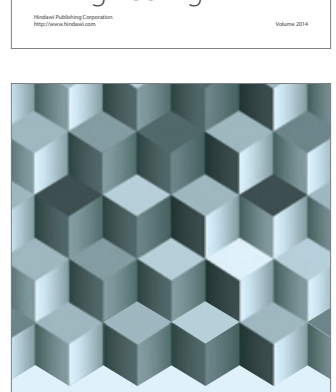

Journal of

Function Spaces
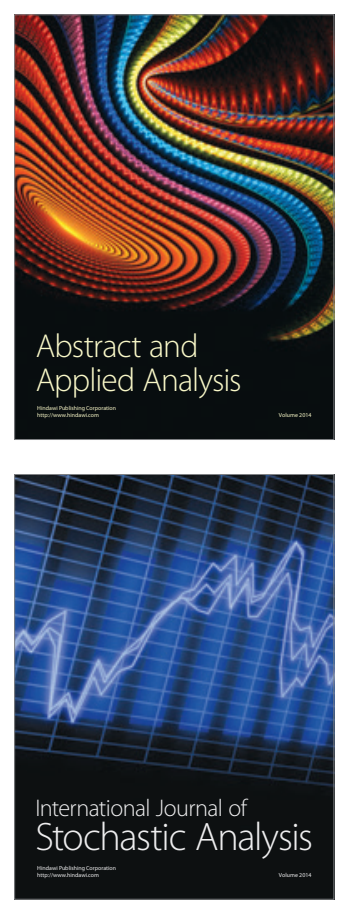

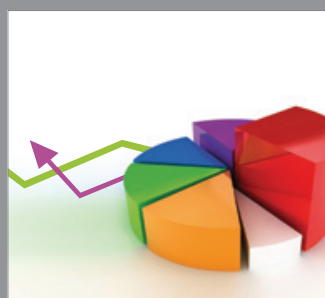

ournal of

Probability and Statistics

Promensencen
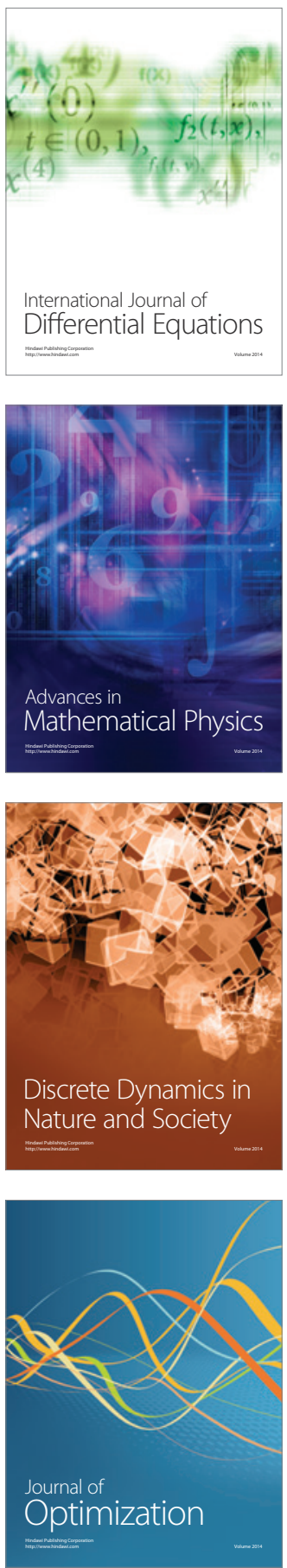